\title{
SPITZER AS A MICROLENS PARALLAX SATELLITE: MASS AND DISTANCE MEASUREMENTS OF BINARY LENS SYSTEM OGLE-2014-BLG-1050L
}

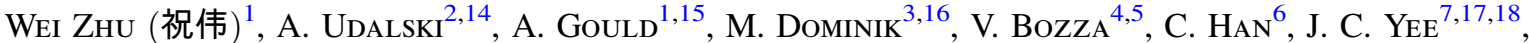 \\ S. Calchi Novati ${ }^{4,8,9,18}$, C. A. Beichman ${ }^{8}$, S. Carey ${ }^{10}$, R. Poleski ${ }^{1,2}$, J. Skowron $^{2}$, S. KozŁowski ${ }^{2}$, P. Mróz ${ }^{2}$, \\ P. Pietrukowicz ${ }^{2}$, G. Pietrzyński ${ }^{2,11}$, M. K. SzYmański ${ }^{2}$, I. Soszyński ${ }^{2}$, K. UlaczyK ${ }^{2}$, Ł. Wyrzykowski ${ }^{2,12}$ \\ (The OGLE Collaboration), \\ AND

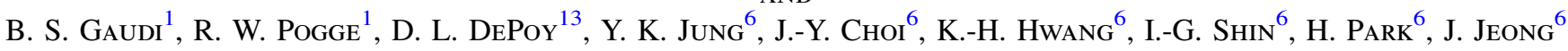 \\ (The $\mu$ FUN COLlaboration) \\ 1 Department of Astronomy, Ohio State University, 140 W. 18th Ave., Columbus, OH 43210, USA \\ 2 Warsaw University Observatory, Al. Ujazdowskie 4, 00-478 Warszawa, Poland \\ 3 SUPA, School of Physics \& Astronomy, North Haugh, University of St Andrews, KY16 9SS, Scotland, UK \\ 4 Dipartimento di Fisica “E. R. Caianiello," Universitá di Salerno, Via Giovanni Paolo II, I-84084 Fisciano (SA), Italy \\ 5 Istituto Nazionale di Fisica Nucleare, Sezione di Napoli, Via Cintia, I-80126 Napoli, Italy \\ ${ }^{6}$ Department of Physics, Institute for Astrophysics, Chungbuk National University, Cheongju 371-763, Korea \\ 7 Harvard-Smithsonian Center for Astrophysics, 60 Garden St., Cambridge, MA 02138, USA
8 NASA Exoplanet Science Institute, MS 100-22, California Institute of Technology, Pasadena, CA 91125, USA \\ 9 Istituto Internazionale per gli Alti Studi Scientifici (IIASS), Via G. Pellegrino 19, I-84019 Vietri Sul Mare (SA), Italy \\ ${ }^{10}$ Spitzer Science Center, MS 220-6, California Institute of Technology, Pasadena, CA 91125, USA \\ ${ }_{11}$ Universidad de Concepción, Departamento de Astronomia, Casilla 160-C, Concepción, Chile \\ 12 Institute of Astronomy, University of Cambridge, Madingley Road, Cambridge CB3 OHA, UK \\ ${ }^{13}$ Department of Physics and Astronomy, Texas A\& M University, College Station, TX 77843-4242, USA \\ Received 2015 January 20; accepted 2015 March 12; published 2015 May 13
}

\begin{abstract}
We report the first mass and distance measurements of a caustic-crossing binary system OGLE-2014-BLG-1050 L using the space-based microlens parallax method. Spitzer captured the second caustic crossing of the event, which occurred $\sim 10$ days before that seen from Earth. Due to the coincidence that the source-lens relative motion was almost parallel to the direction of the binary-lens axis, the fourfold degeneracy, which was known before only to occur in single-lens events, persists in this case, leading to either a lower-mass $\left(0.2\right.$ and $\left.0.07 M_{\odot}\right)$ binary at $\sim 1.1 \mathrm{kpc}$ or a higher-mass $\left(0.9\right.$ and $\left.0.35 M_{\odot}\right)$ binary at $\sim 3.5 \mathrm{kpc}$. However, the latter solution is strongly preferred for reasons including blending and lensing probability. OGLE-2014-BLG-1050 L demonstrates the power of microlens parallax in probing stellar and substellar binaries.
\end{abstract}

Key words: binaries: general - gravitational lensing: micro

\section{INTRODUCTION}

The detection of binary star systems depends on a combination of diverse observational techniques. For example, nearby wide binaries can be directly resolved by highresolution imaging, while close binaries can be detected via eclipsing or spectroscopic methods. To get a full picture of the distributions of the mass ratios, masses, and separations of binary systems, one also needs a technique such as microlensing to probe those binary systems that are difficult for other techniques, such as very low mass binaries (i.e., brown dwarf binaries), dark binaries (e.g., binary black holes), and normal binaries with intermediate separations. For example, two brown dwarf binaries, OGLE-2009-BLG-151/MOA-2009-BLG-232 and OGLE-2011-BLG-0420, were detected via microlensing, with reported total masses of 0.025 and $0.034 M_{\odot}$, respectively (Choi et al. 2013).

The challenge faced by standard microlensing observations is to break the degeneracy between the mass of and the distance

\footnotetext{
14 The OGLE Collaboration.

15 The $\mu$ FUN Collaboration.

${ }_{17}^{16}$ Royal Society University Research Fellow.

17 Sagan Fellow.

18 Sagan Visiting Fellow.
}

to the lens system, since these two physical parameters both enter a single observable quantity-the timescale of the microlensing event, $t_{\mathrm{E}}$ :

$$
t_{\mathrm{E}}=\frac{\theta_{\mathrm{E}}}{\mu_{\text {geo }}} ; \quad \theta_{\mathrm{E}}=\sqrt{\kappa M_{\mathrm{L}} \pi_{\text {rel }}} ; \quad \kappa \equiv \frac{4 G}{c^{2} \mathrm{AU}} \approx 8.14 \frac{\mathrm{mas}}{M_{\odot}} .
$$

Here $\theta_{E}$ is the angular Einstein ring radius, $\mu_{\text {geo }}$ is the geocentric lens-source relative proper motion, and

$$
\pi_{\text {rel }} \equiv \pi_{\mathrm{L}}-\pi_{\mathrm{S}}=\mathrm{AU}\left(\frac{1}{D_{\mathrm{L}}}-\frac{1}{D_{\mathrm{S}}}\right)
$$

is the lens-source relative parallax.

The so-called microlens parallax can in principle be used to meet this challenge (Gould 1992), since the measurement of the microlens-parallax amplitude, $\pi_{\mathrm{E}} \equiv \pi_{\text {rel }} / \theta_{\mathrm{E}}$, directly leads to the determination of lens mass $M_{\mathrm{L}}$ and distance $D_{\mathrm{L}}=\mathrm{AU} / \pi_{\mathrm{L}}$, by

$$
M_{\mathrm{L}}=\frac{\theta_{\mathrm{E}}}{\kappa \pi_{\mathrm{E}}} ; \quad \pi_{\mathrm{L}}=\pi_{\mathrm{E}} \theta_{\mathrm{E}}+\pi_{\mathrm{S}} .
$$

To produce precise constraints on $M_{\mathrm{L}}$ and $D_{\mathrm{L}}$, one therefore needs precise measurements of both $\theta_{\mathrm{E}}$ and $\pi_{\mathrm{E}}$ (keeping in mind that $\pi_{\mathrm{S}}$ is usually known quite accurately). 
Two broad classes of methods have been proposed to measure the microlens parallax $\pi_{\mathrm{E}}$. The first is to obtain observations from a single platform that is being accelerated, which could be Earth (Gould 1992), or a satellite in low-Earth (Honma 1999) or geosynchronous (Gould 2013) orbit. This method has already produced $\sim 100 \pi_{\mathrm{E}}$ measurements (e.g., Alcock et al. 1995; Poindexter et al. 2005; Gaudi et al. 2008). However, it is strongly biased, in the case of binary-lens events, toward nearby lenses (Choi et al. 2013; Jung et al. 2015). The other class of methods is to obtain observations from at least two well-separated observatories (Refsdal 1966; Hardy \& Walker 1995; Gould 1997). In order to produce substantially different light curves observed by different observatories, which lead to precise measurement of $\pi_{\mathrm{E}}$, the required separation between observatories should be $\sim$ AU. For this reason, the so-called terrestrial microlens parallax, i.e., using the ground-based observatories at different sites, only works in very rare cases (Gould et al. 2009; Yee et al. 2009; Gould \& Yee 2015). The combination of ground-based observations and space-based observations from a satellite in solar orbit, called "space-based microlens parallax," has therefore been considered the only way to routinely measure the microlens parallax $\pi_{\mathrm{E}}$ for a substantial fraction of all microlensing events (Refsdal 1966).

There are also several methods proposed to measure the Einstein ring radius $\theta_{\mathrm{E}}$. In principle, $\theta_{\mathrm{E}}$ can be measured by resolving the light centroid of the multiple microlensed images (Walker 1995). However, such a method is currently inaccessible given the fact that the typical Galactic microlensing event has $\theta_{\mathrm{E}} \sim$ mas, although a future space telescope with ultra-precise astrometry may be able to achieve that (Gould \& Yee 2014). The second method is to measure the geocentric lens-source relative proper motion, $\mu_{\text {geo }}$, when the lens and source are well separated, typically a few years before or after the microlensing event occurs, since combining this measurement with $t_{\mathrm{E}}$ also yields $\theta_{\mathrm{E}}$ (Alcock et al. 2001). However, since this method relies on light from both the lens and source, it does not work for a specific class of interesting events, i.e., events with very low mass objects or stellar remnants as lenses. Nevertheless, this approach has been used several times to measure physical parameters of microlensing events (Alcock et al. 2001; Bennett et al. 2006; Dong et al. 2009) and is likely to be used much more frequently in the future (Gould 2014). At present, the most widely exploited method of measuring $\theta_{\mathrm{E}}$ is using the finite source effect that is enabled when the source crosses or closely passes by the caustic structures of the lens system.

What makes binary star systems favorable targets for microlens parallax observations is that the finite source effect is often detected in binary lens events due to their relatively large caustics, producing precise measurement of $\theta_{\mathrm{E}}$ and, if the microlens parallax is also measured, the lens mass $M_{\mathrm{L}}$ and distance $D_{\mathrm{L}}$ (also see Graff \& Gould 2002). It is for this reason that special attention was paid to binary lens events when we were granted Director's Discretionary Time for a $100 \mathrm{hr}$ pilot program to determine the feasibility of using Spitzer as a parallax satellite, although the main objective was to measure lens masses in planetary events (Udalski et al. 2015).

Here we report on the binary lens OGLE-2014-BLG-1050 L, which is the second space-based parallax measurement of a binary lens (Dong et al. 2007) but the first such measurement for a caustic-crossing binary-lens event, or indeed any causticcrossing event. We give a summary of the observations from the ground and Spitzer in Section 2. The light-curve modeling is demonstrated in Section 3; the source and blend characterizations and the derivations of physical parameters are presented in Sections 4 and 5, respectively. In Section 6 we present a discussion of our results.

\section{OBSERVATIONS}

The source star for microlensing event OGLE-2014-BLG1050 lies toward the Galactic bulge field with equatorial and Galactic coordinates (R.A., decl. $)_{2000}=\left(17^{\mathrm{h}} 45^{\mathrm{m}} 07^{\mathrm{s}} .83,-22^{\circ}\right.$ $\left.54^{\prime} 20^{\prime \prime} .0\right)$ and $(l, b)_{2000}=\left(5^{\circ} .09,3^{\circ} .23\right)$, respectively.

It therefore lies just 0.49 above the ecliptic plane.

\subsection{Ground-based Observations}

The Optical Gravitational Lensing Experiment (OGLE) collaboration alerted the community to the new microlensing event OGLE-2014-BLG-1050 on 2014 June $6\left(\mathrm{HJD}^{\prime}=\mathrm{HJD}\right.$ $-2,450,000=6815.3)$, just $35 \mathrm{hr}$ before OGLE detected its first point on the caustic entrance at $\mathrm{HJD}^{\prime}=6816.76$, based on observations with the $1.4 \mathrm{deg}^{2}$ camera on its $1.3 \mathrm{~m}$ Warsaw Telescope at the Las Campanas Observatory in Chile (Udalski 2003).

OGLE-2014-BLG-1050 did not attract much attention when it was initially discovered. This situation changed after early modeling of online OGLE data revealed that it was a binarylens event. Hence, in order to support the Spitzer observations, the Microlensing Follow-Up Network $(\mu \mathrm{FUN})$ started observing this event beginning $\mathrm{HJD}^{\prime}=6822$ using the $1.3 \mathrm{~m}$ SMARTS telescope at CTIO (see Section 2.2). Observations were taken only about once per night in the following few days. For this event, the most crucial task of follow-up teams is to capture the caustic exit with intensive observations so as to constrain $\theta_{\mathrm{E}}$ (see Section 1). However, it is very difficult to predict, based on modeling of the collected data, the exact time of caustic crossing, which normally lasts only a few hours. To achieve that goal, $\mu$ FUN observed at $1 \mathrm{hr}$ cadence and frequently reviewed these data during the night once the light curve began its slow rise toward the caustic exit. The cadence for OGLE-2014-BLG-1050 was increased to $10 \mathrm{hr}^{-1}$ once the caustic exit was recognized.

\subsection{Spitzer Observations}

The general description of the Spitzer observations of this pilot program is given in Udalski et al. (2015). In short, the program was organized into $2.6 \mathrm{hr}$ windows roughly once per day from June $5\left(\mathrm{HJD}^{\prime}=6814\right)$ to July $12\left(\mathrm{HJD}^{\prime}=6851\right)$; observing targets had to be chosen and submitted by J.C.Y. and A.G. to the Spitzer Science Center $\sim 3$ days before the next observing run once per week.

For the particular case of OGLE-2014-BLG-1050, OGLE had not yet issued its alert when the decisions were made for the first week of observations on June $2\left(\mathrm{HJD}^{\prime}=6811.1\right)$. On $\mathrm{HJD}^{\prime}=6817.9$, M.D. suggested that OGLE-2014BLG-1050 might be a binary-lens event, but this was too close to the deadline (6818.1) to modify the observing protocol prior to upload. During the following week, two 


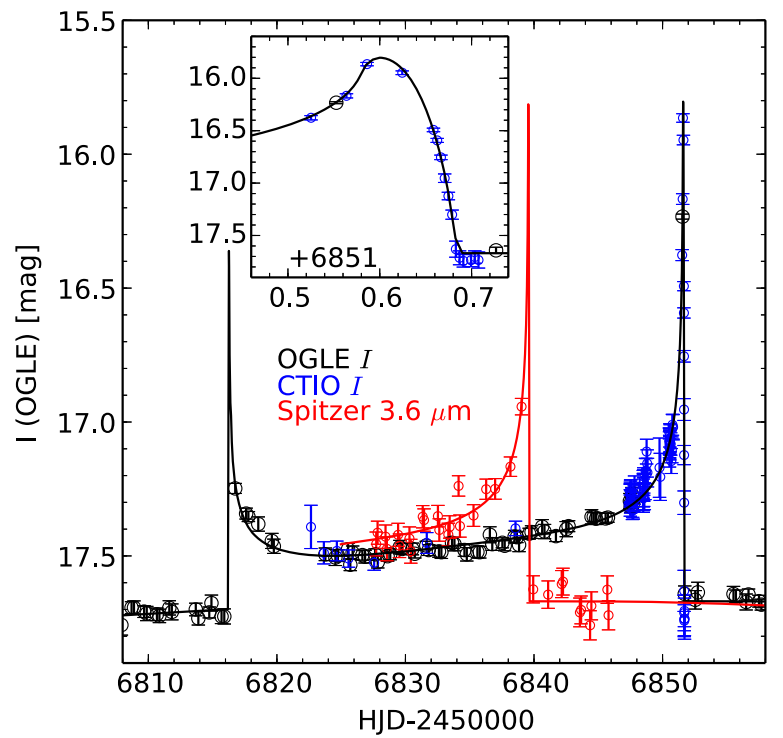

Figure 1. Spitzer's parallax viewpoint of OGLE-2014-BLG-1050, while located $\sim 1$ AU west of Earth. The very broad U-shaped trough in the groundbased light curve indicates a typical caustic-crossing binary-lens event. Spitzer saw the same feature but $\sim 10$ days earlier, suggesting that the lens has a projected velocity of $\sim 200 \mathrm{~km} \mathrm{~s}^{-1}$ due east if Spitzer traces the same trajectory as Earth. The inset shows the details of the caustic exit seen from Earth at $\sim 6851$

modelers (M.D. and V.B.) confirmed it as a binary-lens event, and therefore high priority was assigned to this event, leading to twice-per-day and once-per-day observations in weeks 3 and 4, respectively, and once or twice per day in week 5 until it moved beyond Spitzer's Sun-angle window at $\mathrm{HJD}^{\prime}=6846.56$.

In total, we obtained 31 observations from Spitzer from $\mathrm{HJD}^{\prime}=6828$ to $\mathrm{HJD}^{\prime}=6846$, which also happened to capture the caustic exit of the event. As shown in Figure 1, the Spitzer light curve shows a very similar shape to the ground-based light curve, with the only significant difference being an offset of $\sim 10$ days in time due to the microlens parallax effect, since Spitzer was displaced from the Earth by $\sim 1 \mathrm{AU}$.

\section{LIGHT-CURVE MODELING}

The observed ground-based and space-based light curves of OGLE-2014-BLG-1050 are shown in Figure 1. Only one major feature is noticed in the OGLE data: the prominent $\mathrm{U}$-shaped trough, indicating a typical caustic-crossing binarylens event. The Spitzer data, on the other hand, only captured the caustic exit. The fact that the two light curves show similar shape and almost equal amplitude suggests that the trajectory of the source-lens relative motion as seen from Spitzer should have a similar impact parameter $u_{0}$ to that seen from Earth.

The fourfold degeneracy, coming from the fact that the satellite and Earth can pass on the same side, which we denote as $(+,+)$ and $(-,-)$ solutions with the two signs indicating the signs of $u_{0}$ as seen from Earth and Spitzer, respectively (Gould 2004), or opposite sides of the lens, which we denote as $(+,-)$ and $(-,+)$ solutions accordingly, was well investigated in the case of single-lens microlensing (Refsdal 1966; Gould 1994; Gould \& Horne 2013; Calchi Novati et al. 2014;
Yee et al. 2015). ${ }^{19}$ In the case of binary lenses in which the binary features are detected by both observatories (e.g., Udalski et al. 2015), this fourfold degeneracy normally collapses to the more general twofold degeneracy, namely, $u_{0, \pm}$ degeneracy, whose two solutions give very similar amplitudes of $\pi_{\mathrm{E}}$ and therefore $D_{\mathrm{L}}$ and $M_{\mathrm{L}}$ (Gould \& Horne 2013). However, due to the fact that Spitzer covered only a small portion of the caustic-affected light curve, the persistence of such a fourfold degeneracy is suspected and confirmed in the current binary event.

Considering the characteristics of this event, we adopt a small variant of the standard modeling parameterization. The modeling of the light curves caused by binary lenses usually requires 11 system parameters, including seven basic parameters - the time of the closest lens-source approach, $t_{0}$; the impact parameter normalized by the Einstein radius, $u_{0}$; the Einstein timescale, $t_{\mathrm{E}}$; the normalized source size, $\rho$; the normalized projected separation between the binary components, $s$; the mass ratio, $q$; and the angle from the binary-lens axis to the lens-source relative motion, $\alpha$-and four parameters to allow for higher-order effects, namely, the microlens parallax, quantified by $\pi_{\mathrm{E}, \mathrm{N}}$ and $\pi_{\mathrm{E}, \mathrm{E}}$, and the binary-lens orbital motion, quantified by $d \alpha / d t$ and $d s / d t$. We refer the reader to Udalski et al. (2015) for the sign definitions of parameters $u_{0}, \alpha$, and $d \alpha / d t$. In addition, each observatory requires two flux parameters $\left(f_{\mathrm{S}}, f_{\mathrm{B}}\right)$, so that the total flux is determined by

$$
f_{\text {tot }}(t)=f_{\mathrm{S}} \cdot A(t)+f_{\mathrm{B}},
$$

where $A(t)$ is the magnification of the source as a function of time $t$. These flux parameters are defined in a system in which $f=1$ corresponds to an $18 \mathrm{mag}$ star. For the current event, however, noticing that the time of caustic exit (as observed from Earth), $t_{\mathrm{ce}}$, is much better determined than $t_{0}$, we replace $t_{0}$ with $t_{\mathrm{ce}}$ as a free parameter and then search for the $t_{0}$ that produces the given $t_{\mathrm{ce}}$ in order to compute the light curve (Albrow et al. 1999; Cassan 2008; Kains et al. 2009; Cassan et al. 2010). Parameters leading to an unbound binary system are not taken into account; following Dong et al. (2009), these correspond to models with $\beta>1$, where

$$
\begin{aligned}
& \beta \equiv\left(\frac{E_{\text {kin }}}{E_{\text {pot }}}\right)_{\perp}=\frac{\kappa M_{\odot}(\mathrm{yr})^{2}}{8 \pi^{2}} \frac{\pi_{E} s^{3} \gamma^{2}}{\theta_{E}\left(\pi_{E}+\pi_{\mathrm{S}} / \theta_{E}\right)^{3}} ; \\
& \gamma=\left(\gamma_{\|}, \gamma_{\perp}\right) \equiv\left(\frac{d s / d t}{s}, \frac{d \alpha}{d t}\right) ;
\end{aligned}
$$

and the angular Einstein radius

$$
\theta_{E}=\frac{\theta_{\star, \text { fid }}\left(f_{\mathrm{S}} / f_{\mathrm{S}, \text { fid }}\right)^{1 / 2}}{\rho}
$$

where $\theta_{\star, \text { fid }}$ is the fiducial angular source size that is determined for a fiducial source flux $f_{\text {s.fid }}$ (see Section 4 for the final determination of $\left.\theta_{\star}\right)$.

\footnotetext{
${ }^{19}$ We note that these four solutions are defined in a different way in Yee et al (2015) and Calchi Novati et al. (2014). The four solutions $(+,+),(+,-)$, $(-,-)$, and $(-,+)$ by our definition correspond to $(-,+),(+,+)$, $(-,-)$, and $(+,-)$ solutions by their definition, respectively.
} 
Table 1

Best-fit Parameters for Ground-based-only Fit

\begin{tabular}{lcc}
\hline \hline Parameters & $u_{0}>0($ (“+ Solution) & $u_{0}<0$ (“_” Solution) \\
\hline$\chi^{2} /$ dof & $457.6 / 506$ & $458.4 / 506$ \\
$t_{\mathrm{ce}}\left(\mathrm{HJD}^{\prime}-6851\right)$ & $0.6318 \pm 0.0005$ & $0.6317 \pm 0.0005$ \\
$u_{0}$ & $0.357 \pm 0.014$ & $0.354 \pm 0.017$ \\
$t_{\mathrm{E}}($ days $)$ & $79.2 \pm 6.4$ & $78.0 \pm 4.3$ \\
$\rho\left(10^{-4}\right)$ & $6.09 \pm 0.64$ & $6.23 \pm 0.50$ \\
$\pi_{\mathrm{E}, \mathrm{N}}$ & $-0.45 \pm 0.40$ & $0.36 \pm 0.45$ \\
$\pi_{\mathrm{E}, \mathrm{E}}(\mathrm{deg})$ & $0.31 \pm 0.24$ & $0.40 \pm 0.26$ \\
$\alpha s / d t\left(\mathrm{yr}^{-1}\right)$ & $180.3 \pm 2.4$ & $179.8 \pm 2.3$ \\
$d \alpha / d t\left(\mathrm{yr}^{-1}\right)$ & $\cdots$ & $\cdots$ \\
$s$ & $1.108 \pm 0.028$ & $1.115 \pm 0.023$ \\
$q$ & $0.357 \pm 0.052$ & $0.371 \pm 0.031$ \\
\hline
\end{tabular}

The linear limb-darkening coefficients we adopt, based on the source color, are $\left(\Gamma_{I}, \Gamma_{3.6}\right)=(0.43,0.16)$, following the same procedures as in Udalski et al. (2015).

The Markov chain Monte Carlo (MCMC) method is implemented to find the minimum and the likelihood distribution of parameters. As usual, the point-source, quadrupole, and hexadecapole (Gould 2008; Pejcha \& Heyrovský 2009) approximations are used when the source is approaching, although still reasonably far (a few source radii) from, the caustics. For epochs that are near or on crossing caustics, we use contour integration, in which the limb-darkening effect is accommodated by using 10 annuli (Gould \& Gaucherel 1997; Dominik 1998). ${ }^{20}$ However, this contour integration may fail at some particular points, in which case we use the more time-consuming inverse ray shooting (Dong et al. 2006).

We first fit only the ground-based data, i.e., the $I$-band data from OGLE and CTIO, in order to obtain a first approximate model for this event. The best-fit parameters of the two solutions $\left(u_{0, \pm}\right)$ are listed in Table 1 . Note in particular that the parallax parameters $\pi_{\mathrm{E}, \mathrm{N}}$ and $\pi_{\mathrm{E}, \mathrm{E}}$ are not significantly detected. Because the source lies very close to the ecliptic, the two solutions suffer from the "ecliptic degeneracy" (Jiang et al. 2004; Skowron et al. 2011). The Spitzer data were then included, which yields much better constraints on the microlens parallax vector $\pi_{\mathrm{E}}=\left(\pi_{\mathrm{E}, \mathrm{N}}, \pi_{\mathrm{E}, \mathrm{E}}\right)$. To do so, one needs a careful initial setup of $\pi_{\mathrm{E}, \mathrm{N}}$ and $\pi_{\mathrm{E}, \mathrm{E}}$. That is, the Spitzer caustic crossing clearly occurs between the data points on $\mathrm{HJD}=6839.03$ and $\mathrm{HJD}^{\prime}=6839.94$, so if the trial solutions do not have this property, the $\chi^{2}$ minimization procedure will never arrive there. We have

$$
\pi_{\mathrm{E}}=\frac{\mathrm{AU}}{D_{\perp}}\left(\frac{\Delta t_{0}}{t_{E}}, \Delta u_{0}\right),
$$

in which $\Delta t_{0}=t_{0, \text { sat }}-t_{0, \oplus}, \Delta u_{0}=u_{0, \text { sat }}-u_{0, \oplus}$, and $\boldsymbol{D}_{\perp}$ is the projected separation vector of the Earth and satellite (Udalski et al. 2015). For solutions in which Earth and Spitzer pass the lens on the same side, the similarity between the ground-based and Spitzer light curves suggests that $\Delta u_{0} \approx 0$, which leads to $\pi_{\mathrm{E}, \mathrm{N}} \approx 0$. Then, since $D_{\perp} \approx 1 \mathrm{AU}, \Delta t_{0} \sim 10$

\footnotetext{
20 A more advanced and optimized version of the contour integration proposed by Bozza (2010) has been used in the real-time modeling of data by V.B.
}

days, and $t_{\mathrm{E}} \approx 80$ days, one obtains $\pi_{\mathrm{E}, \mathrm{E}} \approx 0.13$. For the other two solutions, the situation is less straightforward because of the difficulty in determining the direction of $\boldsymbol{D}_{\perp}$ by simple inspection. In principle, one can still estimate $\pi_{\mathrm{E}}$ by working out the geometry more carefully, but a much easier approach is to conduct a grid search on $\left(\pi_{\mathrm{E}, \mathrm{N}}, \pi_{\mathrm{E}, \mathrm{E}}\right)$, with all other parameters initially set at the best fit to ground-based data, so as to find a reasonably good starting point for MCMC sampling. The best-fit parameters for all four solutions of the fit to the combined data sets are listed in Table 2, and the caustic structures and the lens-source relative motion as seen from Earth and Spitzer are shown in Figure 2.

As expected, the inclusion of Spitzer data reduces the error bars on $\pi_{\mathrm{E}, \mathrm{N}}$ and $\pi_{\mathrm{E}, \mathrm{E}}$ significantly. The fourfold degeneracy does persist in this binary-lens event, and the four solutions have nearly equal $\chi^{2}$, mostly due to the coincidence that the source-lens relative motion is almost parallel to the binary-lens direction, i.e., $\alpha \sim 180^{\circ}$, and also the fact that Spitzer data do not have a long enough time baseline. However, this fourfold degeneracy is effectively broken by other considerations, as we will discuss in Section 5.

The inclusion of the lens orbital motion effect is important in order to uncover the true physical parameters of the lens system (Park et al. 2013). For OGLE-2014-BLG-1050, as shown in Table 3 , the orbital motion effect is not significantly detected $\left(\Delta \chi^{2} \approx 5\right)$, but it enlarges the uncertainty on parameters such as $q$ and $u_{0}$ by a factor of 2-3 and therefore has to be taken into account.

It is interesting to compare this binary-lens event with the only planetary event so far found with the same method, OGLE-2014-BLG-0124 (Udalski et al. 2015). One noticeable difference between these two events is the uncertainty in $\pi_{\mathrm{E}}$. In the present binary-lens event, the uncertainty in $\pi_{\mathrm{E}}(10 \%-20 \%)$ after the inclusion of Spitzer data is considerably larger than the one for the planetary event OGLE-2014-BLG-0124 ( 2.5\%). The reason is that the Spitzer light curve of OGLE-2014-BLG1050 has fewer features, which itself derives from two facts. The first is that due to the Sun-angle limitation, Spitzer's total time baseline is not long enough to cover the caustic entrance as was the case for the planetary event. The second is that the current program limits the observation cadence to no more than once per day, and thus we were not able to capture the details of the caustic exit. Another consequence of this singlefeature Spitzer light curve is the asymmetric posterior distributions of $\pi_{\mathrm{E}, \mathrm{N}}$ and $\pi_{\mathrm{E}, \mathrm{E}}$ in the $(+,+)$ and $(-,-)$ solutions. See Figure 3 for the $2 \mathrm{D}$ posteriors of $\left(\pi_{\mathrm{E}, \mathrm{N}}, \pi_{\mathrm{E}, \mathrm{E}}\right)$ from the $(+,+)$ solution with respect to that from the $(+,-)$ solution. This is because Spitzer's view of the source-lens relative trajectory in the $(+,+)[(-,-)]$ solution can go further in the direction of south (north) than north (south). However, this restriction is much weaker for the other two solutions (see Figure 2). We show in the Appendix the triangle diagrams of the fitting parameters and derived physical parameters for the two solutions $(+, \pm)$.

\section{COLOR-MAGNITUDE DIAGRAM}

We characterize the source and "blend" (blending light) here in order to derive the physical parameters of the lens system. The $V-I$ color of the source object is derived from the CTIO instrumental $I-H$ color, and its $I$-band magnitude is determined from the modeling. These color and magnitude are then 


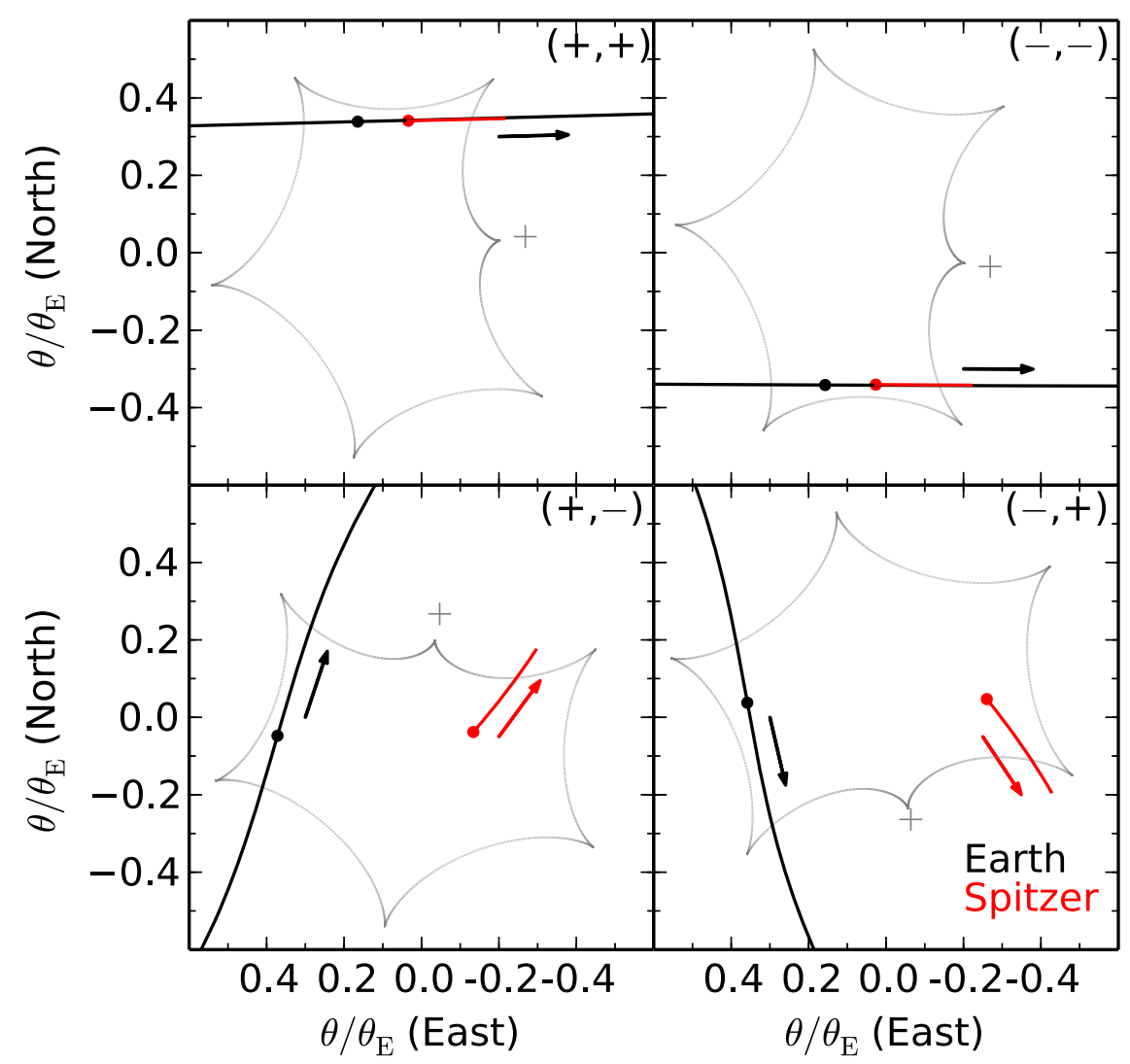

Figure 2. Caustics (gray) and source-lens relative trajectories as seen from Earth (black line) and Spitzer (red line); the red and black dots mark the source positions as seen from Earth and Spitzer when Spitzer started its observations on $\mathrm{HJD}^{\prime} \simeq 6828$ (the size of the dots here does not represent the source size); in each panel, the plus sign marks the position of the primary star, and the secondary is $\sim 1.1$ Einstein units away along the direction of the axis of symmetry and on the opposite side of the caustic. In the favored solutions (upper panels), Spitzer traces the same trajectory as Earth but $\sim 10$ days earlier, resulting in a similar light curve but displaced by $\sim 10$ days (see Figure 1). However, similar light curves could also be induced if Spitzer passed the lens on the opposite side but with similar impact parameters (lower panels). A much larger $\pi_{E}$ is required in these cases, leading to the noticeable wiggles on the source trajectory as seen from Earth.

Table 2

Best-fit Parameters for the Fit to the Combined Data Sets (No Orbital Motion)

\begin{tabular}{|c|c|c|c|c|}
\hline Parameters & $(+,+)$ & $(-,-)$ & $(+,-)$ & $(-,+)$ \\
\hline$\chi^{2 / \mathrm{dof}}$ & $489.5 / 506$ & $489.6 / 506$ & $489.5 / 506$ & $490.4 / 506$ \\
\hline$t_{\mathrm{ce}}\left(\mathrm{HJD}^{\prime}-6851\right)$ & $0.63174 \pm 0.00050$ & $0.63173 \pm 0.00050$ & $0.63157 \pm 0.00049$ & $0.6317 \pm 0.00049$ \\
\hline$u_{0}$ & $0.352 \pm 0.015$ & $0.353 \pm 0.015$ & $0.358 \pm 0.014$ & $0.361 \pm 0.016$ \\
\hline$t_{\mathrm{E}}$ (days) & $76.7 \pm 3.9$ & $76.9 \pm 4.0$ & $77.5 \pm 3.6$ & $77.9 \pm 4.0$ \\
\hline$\rho\left(10^{-4}\right)$ & $6.25 \pm 0.47$ & $6.22 \pm 0.47$ & $6.15 \pm 0.45$ & $6.07 \pm 0.47$ \\
\hline$\pi_{\mathrm{E}, \mathrm{N}}$ & $-0.037 \pm 0.031$ & $0.029 \pm 0.032$ & $-0.577 \pm 0.056$ & $0.571 \pm 0.057$ \\
\hline$\pi_{\mathrm{E}, \mathrm{E}}$ & $0.115 \pm 0.016$ & $0.117 \pm 0.016$ & $0.089 \pm 0.029$ & $0.098 \pm 0.029$ \\
\hline$\alpha(\operatorname{deg})$ & $180.3 \pm 1.6$ & $180.6 \pm 1.6$ & $178.9 \pm 1.8$ & $178.5 \pm 1.7$ \\
\hline$d s / d t\left(\mathrm{yr}^{-1}\right)$ & $\ldots$ & $\ldots$ & $\ldots$ & $\ldots$ \\
\hline$d s / d t\left(\mathrm{yr}^{-1}\right)$ & $\ldots$ & $\ldots$ & $\ldots$ & $\ldots$ \\
\hline$s$ & $1.123 \pm 0.020$ & $1.123 \pm 0.020$ & $1.116 \pm 0.019$ & $1.114 \pm 0.021$ \\
\hline$q$ & $0.382 \pm 0.029$ & $0.380 \pm 0.029$ & $0.383 \pm 0.028$ & $0.383 \pm 0.028$ \\
\hline
\end{tabular}

used to determine the source angular size. We then determine the color and magnitude of the blend in order to consider the possibility that it can be explained by the lens system, by subtracting the source contribution from the total baseline determined by the OGLE IV field star photometry.

We use a variant of the standard procedure to determine the angular size of the source star (Yoo et al. 2004). The standard procedure usually requires magnified images of the event taken in both $V$ and $I$ bands. In the case of OGLE-2014-BLG-1050, $\mu$ FUN did not take any $V$-band images when the source was substantially magnified. Instead, we determine the $V-I$ color from the $I-H$ color following the procedures introduced by Yee et al. (2013), by taking advantage of the fact that the SMARTS camera takes $H$-band images simultaneously with the $I$-band images. The instrumental $I-H$ color of the source is first determined by linear regression of $H$ on the $I$ flux at various magnifications during the event. The $V-I$ color is then found by using the color-color relation derived from 

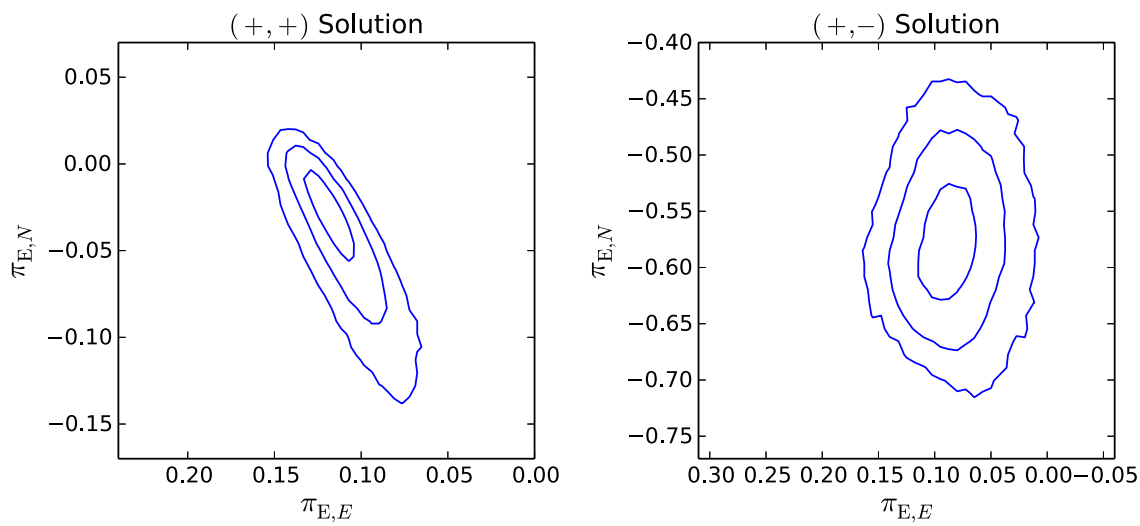

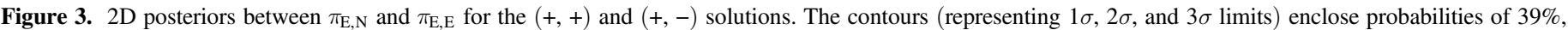
$86 \%$, and $99 \%$, respectively. Compared to the $(+,-)$ solution, the $(+,+)$ solution has much more asymmetric contours in the $\left(\pi_{\mathrm{E}, \mathrm{N}}, \pi_{\mathrm{E}, \mathrm{E}}\right)$ plane.

Table 3

Best-fit Parameters for the Fit to the Combined Data Sets (with Orbital Motion)

\begin{tabular}{|c|c|c|c|c|}
\hline Parameters & $(+,+)$ & $(-,-)$ & $(+,-)$ & $(-,+)$ \\
\hline$\chi^{2} /$ dof & $484.7 / 506$ & $485.4 / 506$ & $483.6 / 506$ & $483.5 / 506$ \\
\hline$t_{0}\left(\mathrm{HJD}^{\prime}-6851\right)$ & $0.63170 \pm 0.00050$ & $0.63176 \pm 0.00048$ & $0.63166 \pm 0.00050$ & $0.63181 \pm 0.00051$ \\
\hline$u_{0}$ & $0.352 \pm 0.036$ & $0.347 \pm 0.030$ & $0.343 \pm 0.034$ & $0.331 \pm 0.033$ \\
\hline$t_{\mathrm{E}}$ (days) & $73.3 \pm 4.2$ & $73.3 \pm 4.2$ & $70.0 \pm 3.8$ & $70.3 \pm 4.3$ \\
\hline$\rho\left(10^{-4}\right)$ & $6.05 \pm 0.64$ & $6.11 \pm 0.59$ & $6.08 \pm 0.65$ & $6.06 \pm 0.58$ \\
\hline$\pi_{\mathrm{E}, \mathrm{N}}$ & $-0.046 \pm 0.051$ & $0.036 \pm 0.036$ & $-0.571 \pm 0.063$ & $0.556 \pm 0.066$ \\
\hline$\pi_{\mathrm{E}, \mathrm{E}}$ & $0.111 \pm 0.025$ & $0.115 \pm 0.020$ & $0.081 \pm 0.035$ & $0.098 \pm 0.031$ \\
\hline$\alpha(\operatorname{deg})$ & $178.8 \pm 2.1$ & $178.8 \pm 2.0$ & $176.9 \pm 2.3$ & $176.5 \pm 2.1$ \\
\hline$d s / d t\left(\mathrm{yr}^{-1}\right)$ & $-0.10 \pm 0.40$ & $-0.19 \pm 0.39$ & $-0.37 \pm 0.49$ & $-0.60 \pm 0.52$ \\
\hline$d \alpha / d t\left(\mathrm{yr}^{-1}\right)$ & $0.57 \pm 0.27$ & $-0.53 \pm 0.27$ & $0.90 \pm 0.53$ & $-0.77 \pm 0.52$ \\
\hline$s$ & $1.113 \pm 0.028$ & $1.113 \pm 0.025$ & $1.105 \pm 0.027$ & $1.097 \pm 0.031$ \\
\hline$q$ & $0.389 \pm 0.094$ & $0.367 \pm 0.078$ & $0.354 \pm 0.093$ & $0.314 \pm 0.090$ \\
\hline
\end{tabular}

nearby field stars. Since nearby field stars are mostly redder than the source, we have to extrapolate the color-color relation blueward to the source position, which introduces a $0.05 \mathrm{mag}$ error in the source $V-I$ color. The instrumental $I$-band baseline flux of the source is determined from the modeling $\left(f_{\mathrm{S}, \text { CTIO }}=0.1612 \pm 0.0016\right)$. Therefore, the instrumental color and magnitude of the source are determined to be $(V-I, I)_{\mathrm{S} \text {. СTIO }}=(-0.73,19.98)$. We then compare the source with the centroid of the red clump and find an offset of $\Delta(V-I, I)_{\mathrm{S}}=(-0.31,3.77)$. With the intrinsic centroid of the red clump (Bensby et al. 2013; Nataf et al. 2013) and a distance modulus of 14.44 (Nataf et al. 2013), we determine the dereddened source color and magnitude to be $(V-I, I)_{\mathrm{S}, 0}=(0.75,18.09)$ and $M_{I}=3.65$, making the source a turn-off star.

To determine the source angular size, we then convert from $V-I$ to $V-K$ using the empirical color-color relations of Bessell \& Brett (1988), ${ }^{21}$ apply the color-surface brightness relation of Kervella et al. (2004), and finally find

$$
\theta_{\star}=0.80 \pm 0.09 \mu \mathrm{as} \text {. }
$$

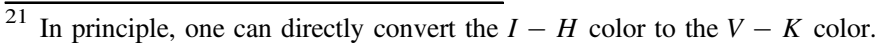
This is not adopted in the present work since the centroid of the red clump is not determined in the $(I, I-H)$ plane as well as in the $(I, V-I)$ plane, and the determination of the red clump centroid in the $(I, I-H)$ plane is not within the scope of the current work.
}

The error comes from the extrapolation of the color-color relation to the source regime $(\sim 0.05 \mathrm{mag})$, the determination of the red clump centroid using nearby field stars $(\sim 0.05 \mathrm{mag})$, and the derivation of the intrinsic source color ( $0.05 \mathrm{mag}$; Bensby et al. 2013).

The light-curve modeling shows that the source is severely blended. Hence, we also determine the color and magnitude of the "blend" (blending light) so as to test whether it can be explained by the lens system. From OGLE IV field star photometry, the "baseline object" (source plus blend) is found to be $(V-I, I)_{\text {base, } \text { OGLE }}=(2.09,17.81)$, and the centroid of the red clump $(V-I, I)_{\mathrm{RC}, \mathrm{OGLE}}=(2.75,16.27)$. The correction of non-standard $V$ band in OGLE IV is then applied,

$$
\Delta(V-I)_{\mathrm{JC}}=0.92 \cdot \Delta(V-I)_{\mathrm{OGLE}},
$$

in which "JC" represents the standard Johnson-Cousins system. With the dereddened red clump at $(V-I, I)_{\mathrm{RC}, \mathrm{JC}}=$ $(1.06,14.32)$ and assuming the same extinction law $R_{I} \equiv$ $A_{I} / E(V-I)=1.23$ (the distance modulus and $R_{V}$ are taken from Nataf et al. 2013, at the event position), the total flux baseline and the source are determined to be $(V-I, I)_{\text {base }, \mathrm{JC}}=$ $(2.04,17.81)$ and $(V-I, I)_{\mathrm{S}, \mathrm{JC}}=(2.34,20.04)$, respectively. We then find the color and magnitude of the blend to be $(V-I, I)_{\mathrm{B}, \mathrm{JC}}=(2.01,17.95)$. 
Table 4

Physical Parameters (Ground-based + Spitzer, with Orbital Motion)

\begin{tabular}{|c|c|c|c|c|}
\hline Parameters & $(+,+)$ & $(-,-)$ & $(+,-)$ & $(-,+)$ \\
\hline$M_{\text {secondary }}\left(M_{\odot}\right)$ & $0.355 \pm 0.079$ & $0.342 \pm 0.092$ & $0.073 \pm 0.016$ & $0.067 \pm 0.013$ \\
\hline$a_{\perp}(\mathrm{AU})$ & $5.02 \pm 0.43$ & $5.04 \pm 0.37$ & $1.64 \pm 0.14$ & $1.66 \pm 0.14$ \\
\hline$\theta_{\mathrm{E}}(\mathrm{mas})$ & $1.34 \pm 0.16$ & $1.32 \pm 0.14$ & $1.33 \pm 0.15$ & $1.32 \pm 0.13$ \\
\hline$\tilde{v}_{\text {hel }, N}\left(\mathrm{~km} \mathrm{~s}^{-1}\right)$ & $-75 \pm 46$ & $58 \pm 46$ & $-42 \pm 4$ & $42 \pm 5$ \\
\hline
\end{tabular}

We show in Figure 4 the color-magnitude diagram (CMD) that is used to characterize the source and blend, after correction to the standard Johnson-Cousins system, together with the color and magnitude of an example lens system from the preferred physical solution (see Section 5).

\section{PHYSICAL PARAMETERS}

The physical parameters derived from our modeling are given in Table 4 , in which $\tilde{\boldsymbol{v}}_{\text {hel }}=\left(\tilde{v}_{\text {hel }, N}, \tilde{v}_{\text {hel, }}\right)$ is the projected velocity of the lens relative to the source in the heliocentric frame, coming from

$$
\tilde{\boldsymbol{v}}_{\mathrm{hel}}=\frac{\boldsymbol{\pi}_{E} \mathrm{AU}}{\pi_{E}^{2} t_{\mathrm{E}}}+\boldsymbol{v}_{\oplus, \perp}
$$

with $\boldsymbol{v}_{\oplus, \perp} \approx(0.7,28.3) \mathrm{km} \mathrm{s}^{-1}$ being the velocity of Earth projected on the sky at the peak of the event. The fourfold degeneracy basically collapses to two physical solutions, since the two solutions $(+,+)$ and $(-,-)$ (collectively $( \pm, \pm)$ ) have the same amplitude of parallax $\pi_{\mathrm{E}}$ (as do the other two solutions $( \pm, \mp)$ ) and therefore lead to similar lens system properties.

Our results show that the microlensing event OGLE-2014BLG-1050 was produced by a binary system consisting of 0.9 and $0.35 M_{\odot}$ stars separated by $5 \mathrm{AU}$ at a distance $3.5 \mathrm{kpc}$ from the Sun (high-mass binary solution), or consisting of 0.2 and $0.07 M_{\odot}$ stars separated by $1.6 \mathrm{AU}$ at a distance $1.1 \mathrm{kpc}$ from the Sun (low-mass binary solution).

The Rich argument, originally suggested by James Rich (circa 1997, private communication) and elaborated in Calchi Novati et al. (2014), argues that if the two components of $\pi_{\mathrm{E}} D_{\perp} / \mathrm{AU}$ (namely, $\Delta t_{0} / t_{\mathrm{E}}$ and $\Delta u_{0}$ ) are small and of the same order, then the $( \pm, \pm)$ solutions are strongly favored over the $( \pm, \mp)$ solutions because the latter require fine-tuning. However, because this argument rests on the axial symmetry of the point-lens geometry, it cannot be applied to binary lenses in a straightforward manner.

As seen in Table 3, the two different physical solutions have nearly equal $\chi^{2}$. However, the color and magnitude of the blend determined in Section 4 strongly support the high-mass binary solution. We demonstrate this point qualitatively by taking a Sun-like primary with a $0.4 M_{\odot}$ secondary at $3.2 \mathrm{kpc}$, all of which are well within the $1 \sigma$ error bar of the high-mass binary solution. The primary would have a color $V-I=0.70$ (Ramírez et al. 2012) and magnitude $M_{I}=4.15$. For the secondary, we take $V-I=2.5$ and $M_{I}=7.6$. Together with the assumed distance $3.2 \mathrm{kpc}$, these give the combined dereddened color and magnitude $(V-I, I)_{\mathrm{L}, 0}=(0.74,16.64)$.

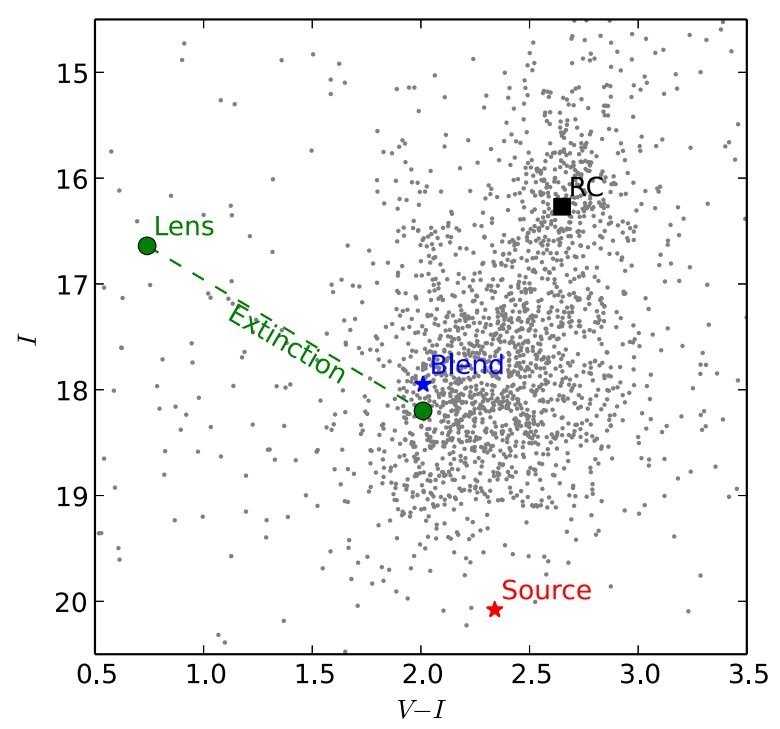

Figure 4. CMD of stars in a $3.8^{\prime} \times 3.8^{\prime}$ square centered on the source star. The positions of the centroid of the red clump ("RC"), the source star, the blended light, and an example lens system taken from the high-mass binary solution before and after the extinction are marked.

Assuming the same $R_{V}$ as we used in Section 4, in order to match the color of the blend, the lens system should suffer an extinction with $E(V-I)=1.27$ and therefore $A_{I}=R_{I} \cdot E(V-I)=1.56$. This leads to an apparent $I$ band magnitude of 18.20 for the lens system. Although it is still 0.25 mag fainter than the total blend, we emphasize that this is just a qualitative demonstration of the consistency between the light-curve modeling and photometry. In fact, many factors could be used to explain this discrepancy, such as metallicity, stellar evolution stage, or a more massive and closer binary system. ${ }^{22}$ We show in Figure 4 this example track of the lens system on the CMD.

In principle, the blend can be a nearby field star other than the lens. However, this scenario is extremely unlikely for this event. Using the OGLE data taken both before and during the event, we are able to independently determine the light centroids of the source and the "baseline object." These give an offset of 42 mas, meaning that the

\footnotetext{
22 Note that a more massive and closer binary system is possible since the error bars on the derived mass and distance are relatively large. For example, raising $\theta_{\mathrm{E}}$ by $5 \%$ increases the mass and relative parallax both by $5 \%$, leading to a roughly $23 \%$ brightness increment.
} 
source of the excess flux must lie within this limit (to be conservative, we use 100 mas for the following estimate). Note that this difference $(0.15$ pixels $)$ is consistent with being coincident because there is a star of comparable brightness to the blend that lies just 0!9 away. With the measured color and magnitude of the excess flux, $(V-I, I)_{\mathrm{B}, \mathrm{JC}}=(2.01,17.95)$, we search for stars whose brightness is within $I_{\mathrm{B}, \mathrm{JC}} \pm 0.5 \mathrm{mag}$ and color within $(V-I)_{\mathrm{B}, \mathrm{JC}} \pm 0.3$, and we find 344 such stars from the CMD shown in Figure 4. Recalling that this CMD is made using all stars within a $3.8 \times 3.8$ square centered on the microlensing event, this indicates that the probability for such a star to lie within 100 mas from the source is only $0.02 \%$.

A complementary argument that also supports the high-mass binary solution is the relative lensing probability. The event rate $\Gamma=n \sigma v$ as a function of the independent physical variables $\left(M_{\mathrm{L}}, D_{\mathrm{L}}, \boldsymbol{\mu}_{\text {rel }}\right)$ for a single-lens microlensing event is (Batista et al. 2011)

$$
\begin{aligned}
p_{\text {single }} & \propto \frac{d^{4} \Gamma}{d D_{\mathrm{L}} d \log M_{\mathrm{L}} d^{2} \boldsymbol{\mu}_{\text {rel }}}=\nu(R, z)\left(2 R_{E}\right) v_{\text {rel }} f\left(\boldsymbol{\mu}_{\text {rel }}\right) g\left(M_{\mathrm{L}}\right) \\
& \propto D_{\mathrm{L}}^{2} \nu(R, z) f\left(\boldsymbol{\mu}_{\text {rel }}\right) M_{\mathrm{L}}^{-\alpha}
\end{aligned}
$$

where $\nu(R, z)$ is the number density of stars at position $(R, z)$ relative to the Galactic center, $R_{\mathrm{E}} \equiv D_{\mathrm{L}} \theta_{\mathrm{E}}$ is the Einstein radius at the lens plane, $v_{\text {rel }} \equiv D_{\mathrm{L}} \mu_{\text {rel }}$ is the lens-source relative velocity, $f\left(\boldsymbol{\mu}_{\text {rel }}\right)$ is the two-dimensional probability function for a given source-lens relative proper motion $\boldsymbol{\mu}_{\text {rel }}$, and $g\left(M_{\mathrm{L}}\right) \equiv M_{\mathrm{L}}^{-\alpha}$ is the Galactic stellar mass function in equal bins of $\log M$. We take $\nu(R, z)$ in the form of

$$
\nu(R, z) \propto e^{-R / R_{\star}} e^{-|z| / H},
$$

and choose $R_{\star} \simeq 3 \mathrm{kpc}$ and $H \simeq 250 \mathrm{pc}$; given the Galactic coordinates of this event, $\nu(R, z)$ yields a factor of 1.4 , favoring the high-mass binary solution. We choose $\alpha=1$ for the power-law index of the mass function. For the relative proper motion, $\mu_{\text {rel }}$ has equal amplitude for each solution, but its direction results in a factor of $\sim 3$ in $f(\boldsymbol{\mu})$, favoring the low-mass binary solution (Calchi Novati et al. 2014; Yee et al. 2015). For a binary-lens event, there should be three factors in addition to those in Equation (4). The multiplicity frequency, $f_{\mathrm{m}}$, for G-type (the primary of the high-mass solution) and Mtype (the primary of the low-mass solution) dwarfs, is different by a factor of $\sim 2$ (Duchêne \& Kraus 2013). The mass ratio $q$ results in a factor of unity, since both solutions have the same $q$. The semimajor axis $a$ also leads to a factor of unity, given an Öpik law in $\log a$ and that $a \simeq \sqrt{3 / 2} a_{\perp}$ (Zhu et al. 2014). With all the above factors, as well as the distance factor $D_{\mathrm{L}}^{2}$, considered, this argument leads to a conclusion that the highmass distant binary is more likely to be microlensed than the low-mass close binary by a factor of $\sim 2$.

The blend origin strongly prefers the high-mass binary solution, and this solution is also preferred by the relative lensing probability. Nevertheless, we suggest that future direct imaging of the event with adaptive optics from ground (e.g., Batista et al. 2014) or from space (e.g., Alcock et al. 2001; Bennett et al. 2006; Dong et al. 2009) will help to reach a definitive conclusion about the nature of the lens system.

\section{DISCUSSION}

After $50 \mathrm{yr}$ of dreaming, the concept originally proposed by Refsdal (1966) to probe the mass function of Galactic astronomical objects without biases toward brightness is finally under way. With combined observations from Spitzer and the ground, we have shown that the mass and distance of the microlensing planetary events can be well constrained (see Udalski et al. 2015), and we have demonstrated the potential of using microlens parallax to probe the Galactic distribution of planets (see Calchi Novati et al. 2014).

In the present work, we report on the binary-lens event OGLE-2014-BLG-1050 observed in our program to demonstrate the power of using space-based microlens parallax to measure the mass and distance of binaries. Binary-lens events attract special attention because the finite source effect is often detected during caustic crossings and thus leads to a wellconstrained Einstein ring radius $\theta_{\mathrm{E}}$.

Unlike the planetary event OGLE-2014-BLG-0124, in which the microlens parallax parameter $\pi_{\mathrm{E}}$ is well determined $(2.5 \%$ uncertainty; Udalski et al. 2015), $\pi_{\mathrm{E}}$ is only constrained to within $\sim 20 \%$ in the present binary-lens event because of its single-feature Spitzer light curve. The caustic entrance was not captured by Spitzer for two reasons. First, by the time of OGLE's alert, the entrance had already occurred as seen by Spitzer. Second, given Spitzer's Sun-angle limitation, it would have been impossible to extend the total time baseline to capture the caustic entrance even with an earlier alert. However, the uncertainty in $\pi_{\mathrm{E}}$ could still have been reduced significantly if more Spitzer observations had been obtained during the caustic exit. Nevertheless, we emphasize that with Spitzer data the measurement of $\pi_{\mathrm{E}}$ is quite secure. By contrast, $\pi_{\mathrm{E}}$ is not significantly detected if only ground-based data are used.

Another interesting characteristic of OGLE-2014-BLG-1050 is that the fourfold degeneracy, which usually appears in singlelens events but has not been investigated in the binary-lens case, is unexpectedly present. This is mostly due to the coincidence that the source-lens relative motion is close to parallel to the binary-lens direction, but also due to the fact that Spitzer data did not capture the caustic entrance. ${ }^{23}$ The resulting four degenerate solutions are almost equal in $\chi^{2}$ and lead to two very different physical solutions for the mass and distance of the lens system: a binary system consisting of 0.9 and $0.35 M_{\odot}$ stars separated by $5 \mathrm{AU}$ at $3.5 \mathrm{kpc}$ (the highmass binary solution), or a binary consisting of 0.2 and $0.07 M_{\odot}$ stars separated by $1.6 \mathrm{AU}$ at $1.1 \mathrm{kpc}$ (the low-mass binary solution).

However, this degeneracy is effectively broken when two other factors are considered. The color and magnitude of the blend, after the source contribution is subtracted from the total baseline, can be well explained by the high-mass binary solution within its $1 \sigma$ error bars. By contrast, the chance that a random field star is responsible for this blend is only $0.02 \%$. The lensing probability estimate also favors the high-mass binary solution by a factor of two.

\footnotetext{
${ }^{23}$ We remind the reader that this fourfold degeneracy will disappear for programs with long enough time baseline.
} 
The microlensing event OGLE-2014-BLG-1050 demonstrates the power of microlens parallax in measuring mass and distance of binaries. Future space-based programs with Spitzer (Gould et al. 2014) and future missions such as Kepler (K2; Gould \& Horne 2013), Euclid (Penny et al. 2013), and the Wild Field InfraRed Survey Telescope (Spergel et al. 2013) can help draw a full picture of the Galactic distribution of binary systems, from brown dwarf binaries to binaries involving black holes.

Work by W.Z., A.G., and B.S.G. was supported by NSF grant AST 1103471. Work by J.C.Y., A.G., and S.C. was supported by JPL grant 1500811 . A.G., B.S.G., and R.W.P. were supported by NASA grant NNX12AB99G. Work by C.H. was supported by the Creative Research Initiative Program (2009-0081561) of the National Research Foundation of Korea. Work by J.C.Y. was performed under contract with the California Institute of Technology (Caltech)/Jet Propulsion Laboratory (JPL) funded by NASA through the Sagan
Fellowship Program executed by the NASA Exoplanet Science Institute. Work by C.A.B. was carried out in part at the Jet Propulsion Laboratory (JPL), California Institute of Technology, under a contract with the National Aeronautics and Space Administration. The OGLE project has received funding from the European Research Council under the European Community's Seventh Framework Programme (FP7/ 2007-2013)/ERC grant agreement no. 246678 to AU. This work is based in part on observations made with the Spitzer Space Telescope, which is operated by the Jet Propulsion Laboratory, California Institute of Technology, under a contract with NASA.

\section{APPENDIX}

We show the triangle diagrams of the fitting parameters for the two solutions $(+,+)$ and $(+,-)$ in Figures 5 and 6 , and those of the derived physical parameters in Figures 7 and 8.

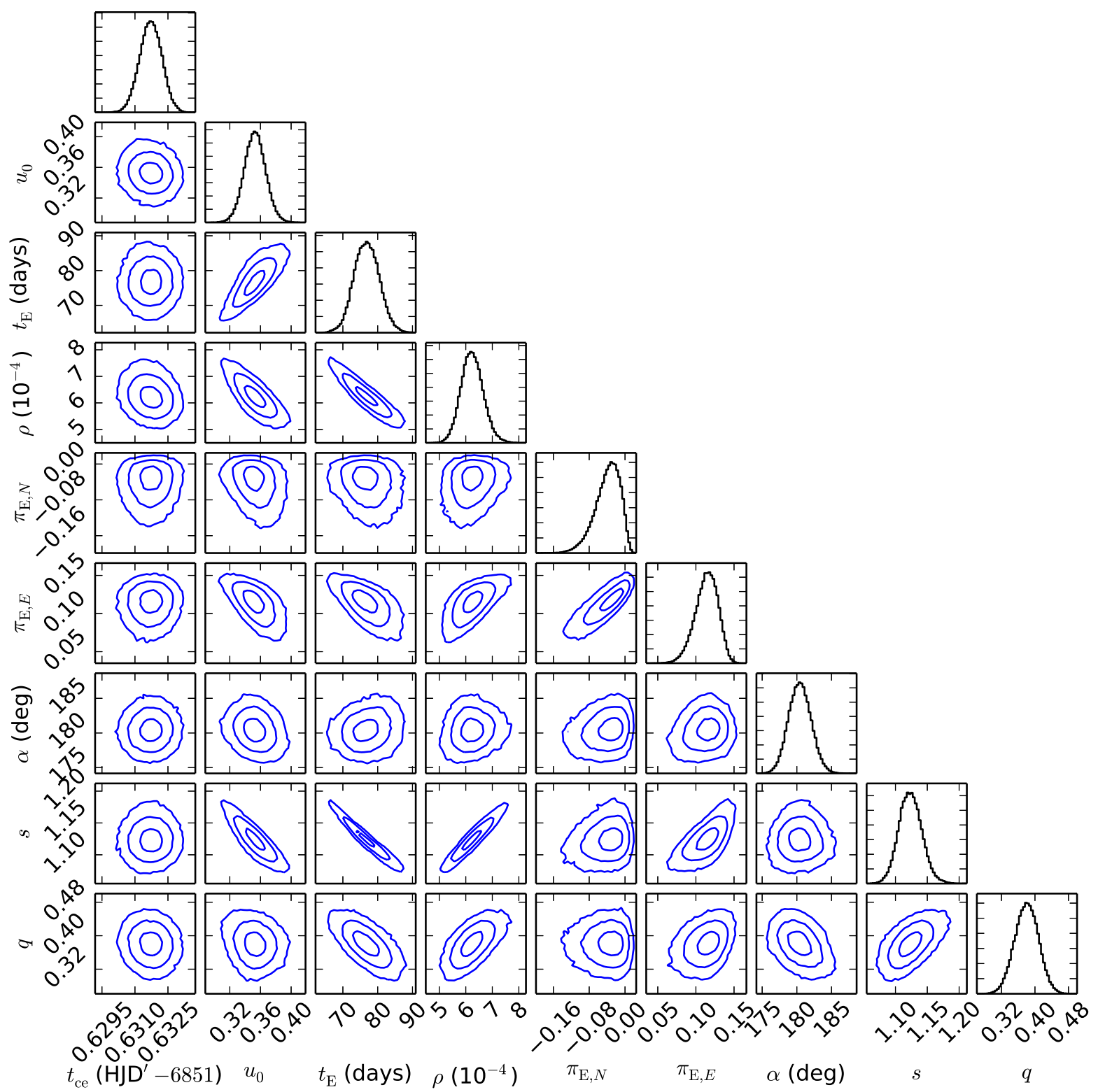

Figure 5. Full triangle diagram of the fitting parameters in the $(+,+)$ solution without orbital motion. The contours (representing $1 \sigma, 2 \sigma$, and $3 \sigma$ limits) enclose probabilities of $39 \%, 86 \%$, and $99 \%$, respectively. 


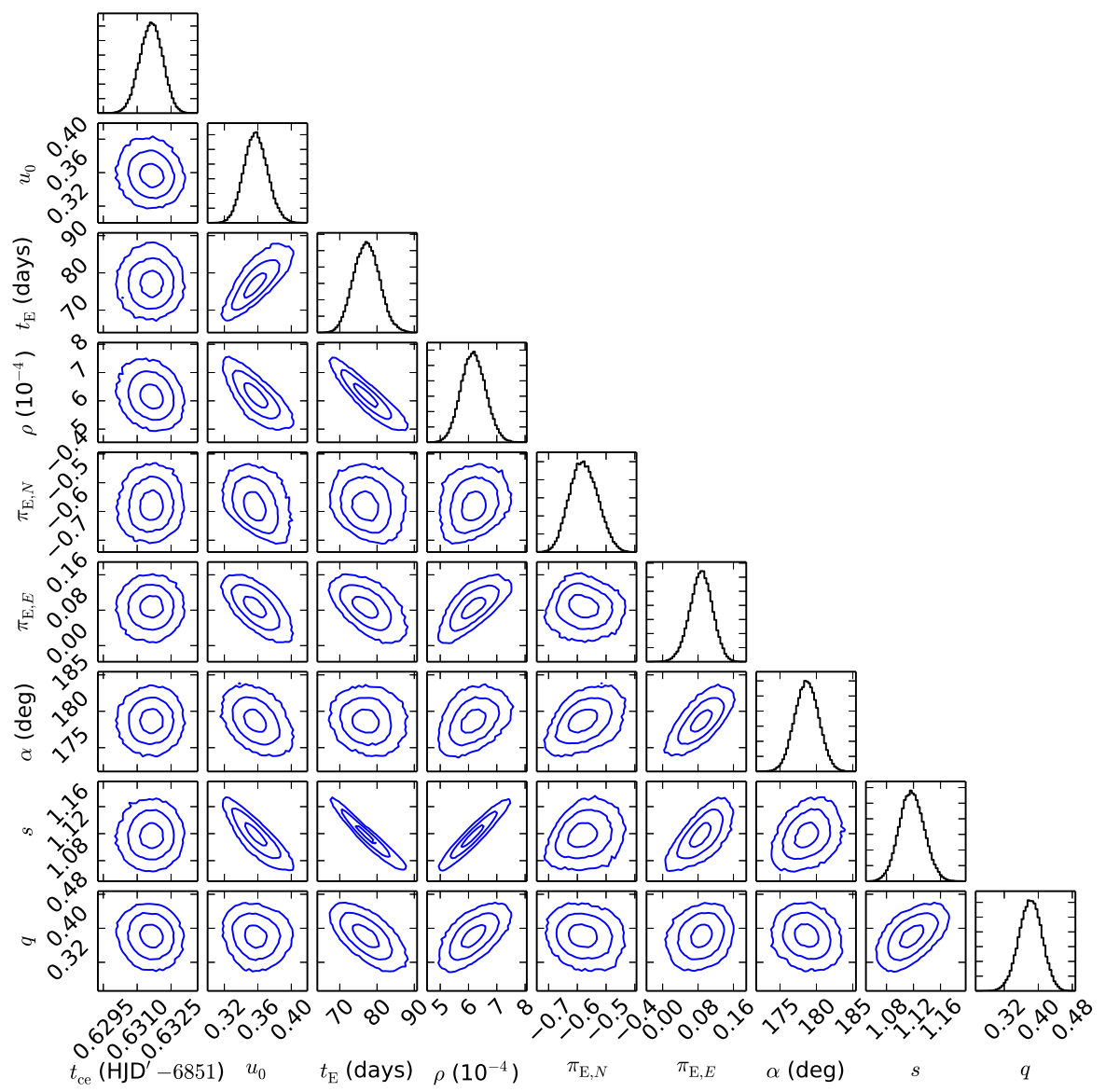

Figure 6. Similar to Figure 5, but for the $(+,-)$ solution.

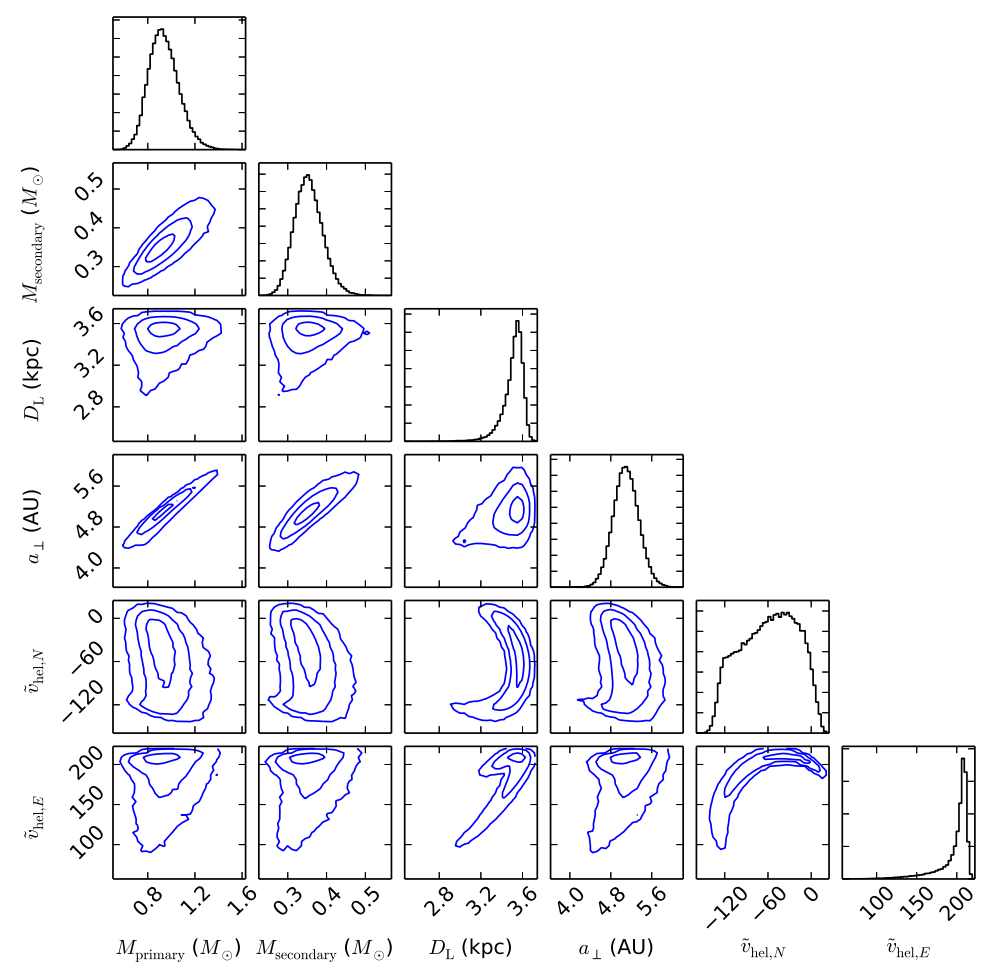

Figure 7. Full triangle diagram of the derived physical parameters in the $(+,+)$ solution without orbital motion. Contours have the same meanings as in Figure 5 . 


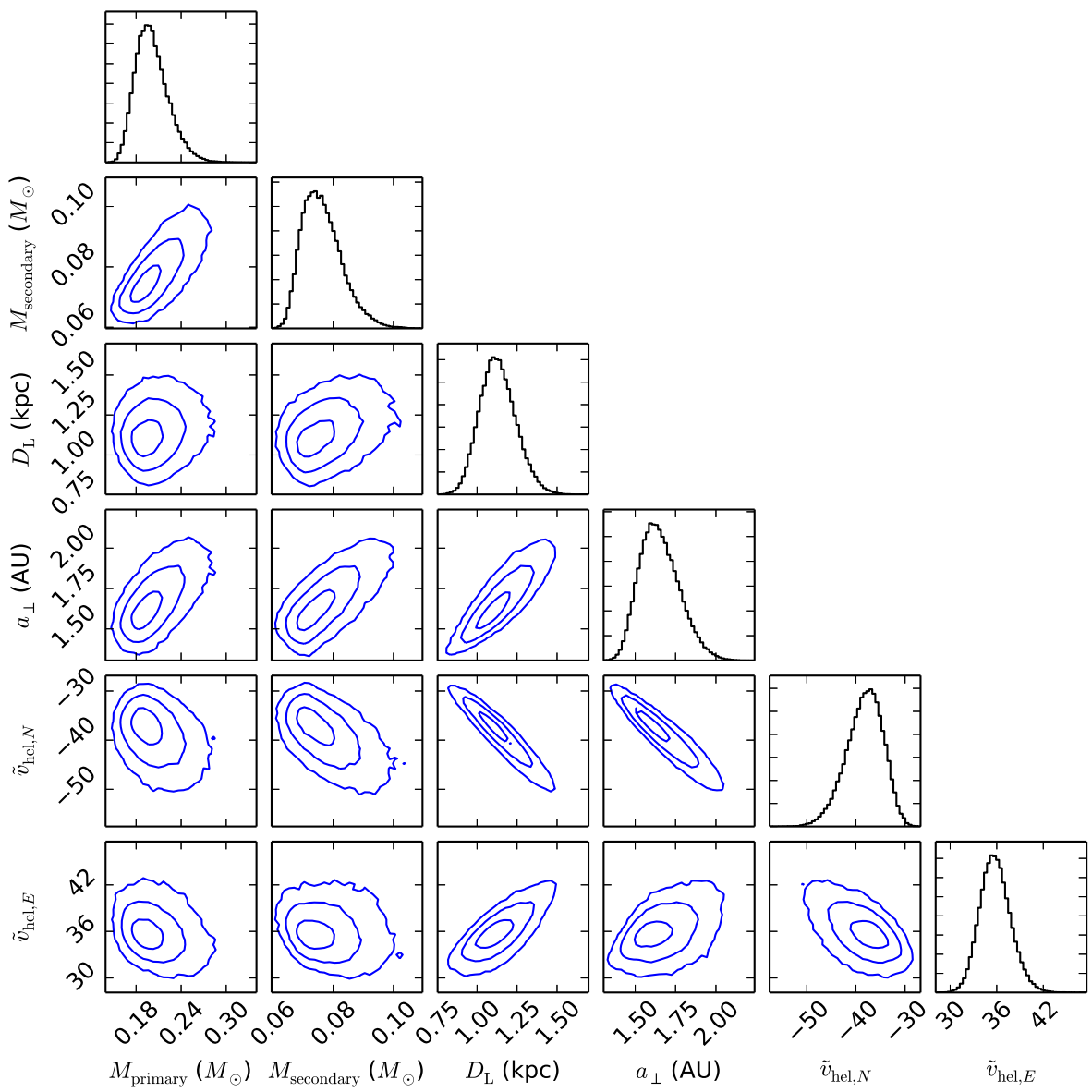

Figure 8. Similar to Figure 7, but for the $(+,-)$ solution.

\section{REFERENCES}

Albrow, M. D., Beaulieu, J.-P., Caldwell, J. A. R., et al. 1999, ApJ, 522, 1022 Alcock, C., Allsman, R. A., Alves, D., et al. 1995, ApJL, 454, L125 Alcock, C., Allsman, R. A., Alves, D. R., et al. 2001, Natur, 414, 617 Batista, V., Beaulieu, J.-P., Gould, A., et al. 2014, ApJ, 780, 54 Batista, V., Gould, A., Dieters, S., et al. 2011, A\&A, 529, A102 Bennett, D. P., Anderson, J., Bond, I. A., Udalski, A., \& Gould, A. 2006, ApJL, 647, L171

Bensby, T., Yee, J. C., Feltzing, S., et al. 2013, A\&A, 549, A147

Bessell, M. S., \& Brett, J. M. 1988, PASP, 100, 1134

Bozza, V. 2010, MNRAS, 408, 2188

Calchi Novati, S., Gould, A., Udalski, A., et al. 2014, arXiv:1411.7378

Cassan, A. 2008, A\&A, 491, 587

Cassan, A., Horne, K., Kains, N., Tsapras, Y., \& Browne, P. 2010, A\&A, 515, A52

Choi, J.-Y., Han, C., Udalski, A., et al. 2013, ApJ, 768, 129

Dominik, M. 1998, A\&A, 333, L79

Dong, S., DePoy, D. L., Gaudi, B. S., et al. 2006, ApJ, 642, 842

Dong, S., Gould, A., Udalski, A., et al. 2009, ApJ, 695, 970

Dong, S., Udalski, A., Gould, A., et al. 2007, ApJ, 664, 862

Duchêne, G., \& Kraus, A. 2013, ARA\&A, 51, 269

Gaudi, B. S., Bennett, D. P., Udalski, A., et al. 2008, Sci, 319, 927

Gould, A. 1992, ApJ, 392, 442

Gould, A. 1994, ApJL, 421, L75

Gould, A. 1997, ApJ, 480, 188

Gould, A. 2004, ApJ, 606, 319

Gould, A. 2008, ApJ, 681, 1593

Gould, A. 2013, ApJL, 763, L35

Gould, A. 2014, JKAS, 47, 215
Gould, A., Carey, S., \& Yee, J. 2014, sptz prop, 11006

Gould, A., \& Gaucherel, C. 1997, ApJ, 477, 580

Gould, A., \& Horne, K. 2013, ApJL, 779, L28

Gould, A., Udalski, A., Monard, B., et al. 2009, ApJL, 698, L147

Gould, A., \& Yee, J. C. 2012, ApJL, 755, L17

Gould, A., \& Yee, J. C. 2014, ApJ, 784, 64

Graff, D. S., \& Gould, A. 2002, ApJ, 580, 253

Hardy, S. J., \& Walker, M. A. 1995, MNRAS, 276, L79

Honma, M. 1999, ApJL, 517, L35

Jiang, G., DePoy, D. L., Gal-Yam, A., et al. 2004, ApJ, 617, 1307

Jung, Y. K., Udalski, A., Sumi, T., et al. 2015, ApJ, 798, 123

Kains, N., Cassan, A., Horne, K., et al. 2009, MNRAS, 395, 787

Kervella, P., Thévenin, F., di Folco, E., \& Ségransan, D. 2004, A\&A, 426, 297

Nataf, D. M., Gould, A., Fouqué, P., et al. 2013, ApJ, 769, 88

Park, H., Udalski, A., Han, C., et al. 2013, ApJ, 778, 134

Pejcha, O., \& Heyrovský, D. 2009, ApJ, 690, 1772

Penny, M. T., Kerins, E., Rattenbury, N., et al. 2013, MNRAS, 434, 2

Poindexter, S., Afonso, C., Bennett, D. P., et al. 2005, ApJ, 633, 914

Ramírez, I., Michel, R., Sefako, R., et al. 2012, ApJ, 752, 5

Refsdal, S. 1966, MNRAS, 134, 315

Spergel, D., Gehrels, N., Breckinridge, J., et al. 2013, arXiv:1305.5422

Skowron, J., Udalski, A., Gould, A., et al. 2011, ApJ, 738, 87

Udalski, A. 2003, AcA, 53, 291

Udalski, A., Yee, J. C., Gould, A., et al. 2015, ApJ, 799, 237

Walker, M. A. 1995, ApJ, 453, 37

Yee, J. C., Hung, L.-W., Bond, I. A., et al. 2013, ApJ, 769, 77

Yee, J. C., Udalski, A., Calchi Novati, S., et al. 2015, ApJ, 802, 76

Yee, J. C., Udalski, A., Sumi, T., et al. 2009, ApJ, 703, 2082

Yoo, J., DePoy, D. L., Gal-Yam, A., et al. 2004, ApJ, 603, 139

Zhu, W., Penny, M., Mao, S., Gould, A., \& Gendron, R. 2014, ApJ, 788, 73 